\title{
The protective effects of GYY4137 on ipsilateral testicular injury in experimentally varicocele-induced rats
}

\author{
JIN-ZHUO NING ${ }^{1 *}$, WEI LI ${ }^{2 *}$, FAN CHENG $^{1}$, TING RAO $^{1}$, WEI-MIN YU ${ }^{1}$, YUAN RUAN $^{1}$, \\ RUN YUAN $^{1}$, XIAO-BIN ZHANG ${ }^{1}$, YANG DU ${ }^{1}$ and CHENG-CHENG XIAO ${ }^{1}$ \\ Departments of ${ }^{1}$ Urology and ${ }^{2}$ Anesthesia, Renmin Hospital of Wuhan University, Wuhan, Hubei 430060, P.R. China
}

Received June 14, 2017; Accepted September 1, 2017

DOI: 10.3892/etm.2017.5417

\begin{abstract}
The aim of the present study was to evaluate whether morpholin-4-ium 4 methoxyphenyl (morpholino) phosphonodithioate (GYY4137) exhibits a protective effect on ipsilateral testicular injury in experimentally varicocele (VC)-induced rats. A total of 48 rats were randomly divided into the following 6 groups ( $\mathrm{n}=8$ each): Group A (control group); group B (sham group); group C (VC group); group D (VC group administered $5 \mathrm{mg} / \mathrm{kg} /$ day GYY4137); group E (VC group administered $10 \mathrm{mg} / \mathrm{kg} /$ day GYY4137) and group F (VC group administered $20 \mathrm{mg} / \mathrm{kg} /$ day GYY4137). Indicators of oxidative stress, apoptosis and inflammation were measured to evaluate the effect of GYY4137 on ipsilateral testicular injury. Compared with groups $\mathrm{A}$ and $\mathrm{B}$, rats in group $\mathrm{C}$ exhibited severe histological changes and an increase in oxidative stress, apoptosis and inflammation. By contrast, amelioration of testicular damage was evident in the group D, E and F that were treated with GYY4137. These results demonstrate that GYY4137 may be a promising therapy to treat $\mathrm{VC}$ as it alleviates oxidative stress, apoptosis and inflammation in experimentally VC-induced rats.
\end{abstract}

\section{Introduction}

A varicocele (VC) is defined as the abnormal expansion, elongation and tortuosity of the spermatic vein and is considered to be the primary cause of infertility in males (1). The exact mechanism of testicular dysfunction and infertility induced by VC has not yet been clearly defined. It may be associated with a number of factors, including microcirculation disturbance of the testis, vasoactive substance reflux, oxidative stress, an

Correspondence to: Dr Fan Cheng, Department of Urology, Renmin Hospital of Wuhan University, 238 Jiefang Road, Wuhan, Hubei 430060, P.R. China

E-mail: chenfan_93@126.com

*Contributed equally

Key words: GYY4137, varicocele, oxidative stress, apoptosis, inflammation increase in nitric oxide (NO) concentration, hypoxia, hyperthermia, inflammation or apoptosis (2). It has been reported that the excessive production of reactive oxygen species (ROS) causes germinal cell necrosis, endothelial damage and DNA damage, leading to sperm dysfunction in VC-induced rats (3). Furthermore, elevated spermatogenic cell apoptosis and testicular inflammation are closely associated with male infertility (4).

Hydrogen sulfide $\left(\mathrm{H}_{2} \mathrm{~S}\right)$ is a colourless, water-soluble, volatile gas with a characteristic smell of rotten eggs and was initially considered to be a toxic gas (5). It has been suggested that $\mathrm{H}_{2} \mathrm{~S}$ may be a third type of endogenous gaseous transmitter, such as carbon monoxide (CO) and NO, and may induce antioxidant, anti-inflammatory and anti-apoptotic effects in various systems $(6,7)$. NaHS has been widely used in clinical experiments to evaluate the biological effects of $\mathrm{H}_{2} \mathrm{~S}(5,8)$. However, NaHS releases large amounts of $\mathrm{H}_{2} \mathrm{~S}$ over a short period of time and cannot effectively imitate the biological course of naturally produced $\mathrm{H}_{2} \mathrm{~S}$ (9). Morpholin-4-ium 4 methoxyphenyl (morpholino) phosphonodithioate (GYY4137) was one of a series of compounds synthesized that were based on the chemical structure of Lawesson's compound, which releases $\mathrm{H}_{2} \mathrm{~S}$ in organic solvents (10). GYY4137 is novel $\mathrm{H}_{2} \mathrm{~S}$ donor that, unlike NaHS, decomposes and over a period of a few hours produces small amounts of $\mathrm{H}_{2} \mathrm{~S}$ under physiological conditions, thus potentially mimicking $\mathrm{H}_{2} \mathrm{~S}$ release in vitro and in vivo (10). Meng et al (11) reported that GYY4137 may significantly increase the ventricular ejection fraction and reduce the ischemic area in rats, thus protecting against myocardial ischemia-reperfusion injury by attenuating oxidative stress and apoptosis. Additionally, Tang et al (12) demonstrated that GYY4137 serves an anti-inflammatory role in the process of acute lung injury in rats. Therefore, the current study hypothesized that GYY4137 has the ability to ameliorate oxidative stress, inflammation and spermatogenic cell apoptosis.

\section{Materials and methods}

Animals. The current study involved 48 adult male Sprague Dawley rats (8-10 weeks), weighing 250-300 g, obtained from the Hubei Center for Disease Control (Hubei, China). Prior to the experiment, rats had free access to food and water in a $12 \mathrm{~h}$ light/dark cycle with a constant temperature $\left(22 \pm 2^{\circ} \mathrm{C}\right)$ and humidity (40-70\%). All experimental procedures adhered to 
the National Institutes of Health Guide for the Care and Use of Laboratory Animals (13) and were approved by the Animal Care and Use Committee of Wuhan University (Wuhan, China).

Experimental grouping and surgical procedures. All rats were anaesthetized by intraperitoneal administration of $2 \%$ sodium phenobarbital $(50 \mathrm{mg} / \mathrm{kg}$; Propbs Biotechnology. Co., Ltd., Beijing, China) and were then placed on a homoeothermic table to maintain a rectal temperature of $37-38^{\circ} \mathrm{C}$. The 48 rats were then randomly divided into 6 different groups (all $n=8$ ). The rats in group A (control) did not undergo surgery. The rats in group B (sham group) underwent an abdominal median incision. The left renal vein was separated and there was no additional intervention. The rats in group C (VC group), underwent a procedure in which the left renal vein was carefully separated on the inside of the adrenal vein and the spermatic vein, and a metal probe $(0.5-0.85 \mathrm{~mm}$ diameter) was placed parallel to the left renal vein. The vein and metal probe were ligated together with 4-0 silk suture, reducing the vein diameter to $\sim 50 \%$ of its original diameter. The metal probe was then removed and the left renal vein was reversibly constricted. This procedure followed the procedure previously performed by Turner (14) and induced the following characteristics in the rats: Abnormal expansion, elongation and tortuosity of the left renal and spermatic vein; diameter of the left renal vein $>1 \mathrm{~mm}$ and no pathological lesion in the left kidney. The rats in groups $\mathrm{D}, \mathrm{E}$ and $\mathrm{F}$ underwent the procedures performed in group $\mathrm{C}$ followed by the intraperitoneal administration of 5, 10 or $20 \mathrm{mg} / \mathrm{kg}$ GYY4137 (Sigma-Aldrich; Merck KGaA, Darmstadt, Germany), once a day respectively.

A total of 4 weeks following the successful establishment of the $\mathrm{VC}$ model, the left testis of the rat from the all groups was removed and divided into two halves. One half was fixed in $4 \%$ paraformaldehyde at $4^{\circ} \mathrm{C}$ for $12 \mathrm{~h}$ prior to histological examination. The other half was immediately frozen at $-80^{\circ} \mathrm{C}$ for later analysis.

Hematoxylin and eosin $(H \& E)$ staining. Following fixation in $4 \%$ paraformaldehyde, the testis tissue was embedded in paraffin and cut into $4-\mu \mathrm{m}$ thick sections following standard protocols. Following routine dewaxing and hydrating, sections were stained with hematoxylin for $5 \mathrm{~min}$ and eosin for $2 \mathrm{~min}$ at the room temperature.

Biochemical evaluation. Frozen testicular tissues were homogenized and centrifuged at $500 \mathrm{xg}$ for $10 \mathrm{~min}$ at $4^{\circ} \mathrm{C}$, and the supernatants were collected. Testicular Malondialdehyde (MDA) content and superoxide dismutase (SOD) activity were measured spectrophotometrically using thiobarbituric acid (TBA) and xanthine oxidase methods following the manufacturer's protocol (A003-1; Malondialdehyde assay kit; Jiancheng Bioengineering Institute, Nanjing, China). MDA content was detected by measuring absorbance at $532 \mathrm{~nm}$ and expressed as nmol/g protein. SOD activity was determined by measuring absorbance at $550 \mathrm{~nm}$ using a microplate reader and presented as $\mathrm{U} / \mathrm{mg}$ protein.

TUNEL assays. A TUNEL assay was performed to detect spermatogenic cell apoptosis in the testes using the transferase-mediated dUTP nick-end labelling (TUNEL) method with an in situ apoptosis detection kit (Roche Diagnostics, Basel, Switzerland) following the manufacturer's protocol. Briefly, the rat testis tissue was fixed in $4 \%$ paraformaldehyde/PBS solution ( $\mathrm{pH} 7.4)$ at $4{ }^{\circ} \mathrm{C}$ overnight and then placed into $70 \%$ ethanol at $20^{\circ} \mathrm{C}$ for $24 \mathrm{~h}$. Following washing 3 times with PBS, the samples were immersed in a permeabilization buffer for $15 \mathrm{~min}$. Subsequently, they were incubated with $50 \mathrm{ml}$ reaction buffer (TdT Enzyme $5 \mathrm{ml}$ + Labeling Safe Buffer $45 \mathrm{ml}$ ) at $37^{\circ} \mathrm{C}$ for $90 \mathrm{~min}$. Nuclei that stained brown were considered to be TUNEL-positive cells as observed under a Zeiss LSM 510 confocal laser scanning microscope (Zeiss AG, Oberkochen, Germany). Five high-power fields of vision were randomly selected in each slice and the average number of apoptotic cells per 200 cells was calculated. The apoptosis index (AI) was expressed as follows: $\mathrm{AI}=$ (positive cells/total cells counted) x $100 \%$.

Immunohistochemistry. Caspase-3 and Bax expression were measured using immunohistochemical staining. Tissues were fixed in $4 \%$ paraformaldehyde, embedded in paraffin and then cut in $4 \mu \mathrm{m}$ thickness, and endogenous peroxidase activity was blocked with $3 \%$ hydrogen peroxide at $37^{\circ} \mathrm{C}$ for $10 \mathrm{~min}$. The sections were incubated with $10 \%$ normal goat serum in Tris-buffered saline (TBS) for $30 \mathrm{~min}$ at $37^{\circ} \mathrm{C}$. Staining was performed using rabbit polyclonal anti-caspase-3 (sc7148; Santa Cruz Biotechnology, Inc., Dallas, TX, USA) and rabbit polyclonal anti-Bax (sc493; Santa Cruz Biotechnology, Inc.) antibodies. After being washed three times with PBS, all sections were incubated in DAB reagents and counterstained with haematoxylin. All steps were performed following the manufacturer's instructions and the results were evaluated by comparing the staining intensity with an Olympus BX50 light microscope (Olympus Corporation, Tokyo, Japan).

Western blot analysis. All testicular tissue proteins were extracted with radioimmunoprecipitation assay lysis buffer (P0013B; Beyotime Institute of Biotechnology, Haimen, China) then quantified using a bicinchoninic acid assay. Briefly, $40 \mu \mathrm{g} /$ lane protein samples were separated by $10 \%$ SDS-PAGE and transferred to a polyvinylidene difluoride membrane. The membrane was blocked at $37^{\circ} \mathrm{C}$ for $2 \mathrm{~h}$ with $5 \%$ non-fat milk in TBST buffer and then incubated with the following primary antibodies at $4^{\circ} \mathrm{C}$ overnight: Bax (sc-493; 1:500; Santa Cruz Biotechnology, Inc.), cleaved-caspase-3 (sc7148; 1:1,000; Santa Cruz Biotechnology, Inc.), tumor necrosis factor (TNF)- $\alpha$ (ab6671; 1:500; Abcam, Cambridge, UK) and interleukin (IL)-1 $\beta$ (ab100768; 1:200; Abcam). Following three washes with TBST buffer, membranes were incubated with secondary goat anti-rabbit antibodies conjugated with horseradish peroxidase (LK2001/LK2003; 1:100; Sungene Biotech, Co., Ltd., Tianjin, China) at room temperature for $1 \mathrm{~h}$. All specific bands were visualized using an enhanced chemiluminescence system (Pierce; Thermo Fisher Scientific, Inc., Waltham, MA, USA). Optical densities were detected using ImageJ software version 1.48 (National Institute of Health, Bethesda, MD, USA).

Reverse transcription-quantitative polymerase chain reaction $(R T-q P C R)$. Total RNA was extracted from the testicular tissue 


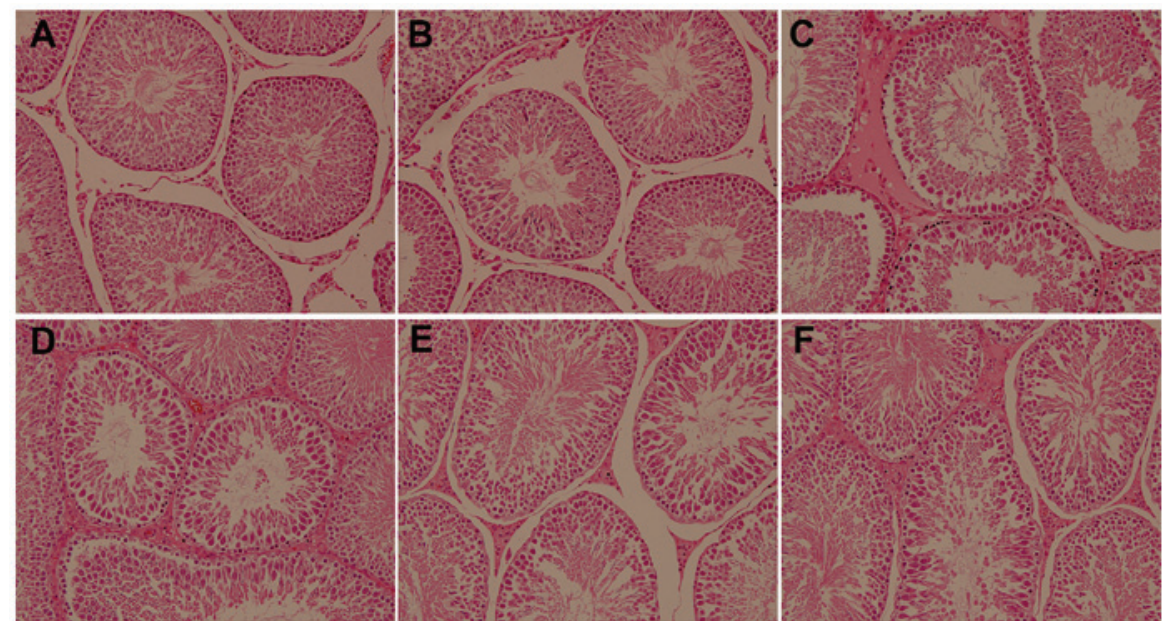

Figure 1. Microphotographs of testes tissues in the 6 groups. (A) Control group, (B) sham group, (C) VC group, (D) VC+5 mg/kg/day GYY4137 group, (E) VC+10 mg/kg/day GYY4137 group and (F) VC+20 mg/kg/day GYY4137 group. Groups A and B exhibited no marked morphological changes. Group C indicated significant pathological and morphological changes. Groups D, E and F exhibited the reversal of these changes. Hematoxylin and eosin staining; magnification, x200. VC, varicocele; GYY4127, Morpholin-4-ium 4 methoxyphenyl (morpholino) phosphonodithioate.

samples from each group using TRIzol reagent (Invitrogen; Thermo Fisher Scientific, Inc.) and RNA concentration was detected using spectrophotometry. First-strand cDNA was synthesized using a cDNA synthesis kit (Promega Corporation, Madison, WI, USA), following the manufacturer's protocol. Subsequently, qPCR was performed using an Applied Biosystems SYBR Green mix kit on an ABI 7900 Real-Time PCR system (Applied Biosystems; Thermo Fisher Scientific, Inc.). The primer sequences for TNF- $\alpha$ and IL-1 $\beta$ were as follows: TNF- $\alpha$ forward, 5'-ATCCGCGACGTGGAACTG-3', and reverse, 5'-ACCGCCTGGAGTTCTGGAA-3'; IL-1 $\beta$ forward, 5'-GAGCACCTTCTTTTCCTTCATCTT-3', and reverse, 5'-TCACACACCAGCAGGTTATCATC-3'. GAPDH was used as a housekeeping gene and the primer sequences for GAPDH were as follows: Forward, 5'-ACAGCAACAGGG TGGTGGAC-3' and reverse, 5'-TTTGAGGGTGCAGCGAAC TT-3'. The data were presented as a ratio to GAPDH mRNA. qPCR was performed with 40 cycles of $94^{\circ} \mathrm{C}$ for $30 \mathrm{sec}$, followed by $56^{\circ} \mathrm{C}$ for $30 \mathrm{sec}$ and $72^{\circ} \mathrm{C}$ for $25 \mathrm{sec}$. The quantitative analysis was conducted using the $2^{-\Delta \Delta \mathrm{Cq}}$ method (15).

Statistical analysis. All data are presented as the mean \pm standard deviation. Statistical analysis was performed using SPSS 19.0 (IBM Corp, Armonk, NY, USA). The means were compared using one-way analysis of variance followed by the Student-Newman-Keuls test for the different groups. $\mathrm{P}<0.05$ was considered to indicate a statistically significant difference. All experiments were performed at least 3 times.

\section{Results}

GYY4137 alleviates VC-induced histopathological damage. $\mathrm{H} \& \mathrm{E}$ staining indicated that there were no marked morphological changes in the left testicular tissue of rats in groups A and $\mathrm{B}$. The spermatogenic cells in the seminiferous tubules were arranged in order, including the primary and secondary spermatocytes, spermatids and spermatozoa. Rats in group C exhibited marked damage in their spermatogenic function, as determined by the detachment of epithelial cells in the lumen, the disordered arrangement of spermatogenic cells and extensive damage of the seminiferous epithelium. However, treatment with GYY4137 reduced severe testicular damage and there were fewer spermatogenic cells and seminiferous epithelium changes in group $\mathrm{C}$ compared with groups D, E and F (Fig. 1).

GYY4137 reduces the increases in MDA content and reduction in SOD activity induced by VC. To evaluate levels of oxidative stress in VC-induced rats, MDA content and SOD activity were measured in testicular tissue. In group C, MDA content was significantly increased and SOD activity was significantly decreased, compared with groups A and B. However, treatment GYY4137 significantly reversed the increase in MDA content and the reduction in SOD activity induced by VC in groups D, E and F (Fig. 2).

GYY4137 inhibits the apoptosis of spermatogenic cells induced by $V C$. To assess apoptosis and determine the AI, the expression of Bax and caspase- 3 in testicular spermatogenic cells was evaluated by TUNEL, immunohistochemistry and Western blot analysis. There was a significant increase in the number of TUNEL-positive spermatogenic cells in group $\mathrm{C}$ compared with groups A and B (Fig. 3). Furthermore, immunohistochemistry indicated that caspase- 3 and Bax levels were markedly increased in group $\mathrm{C}$ compared with groups $\mathrm{A}$ and B. However, following treatment with GYY4137, significantly fewer TUNEL-positive spermatogenic cells were detected. Additionally, treatment with GYY4137 decreased caspase-3 and Bax expression. Testis taken from mice in group $\mathrm{E}$ that received $10 \mathrm{mg} / \mathrm{kg} /$ day GYY4127 experienced an ideal therapeutic effect on damaged testes, evident by a significant reduction of TUNEL-positive spermatogenic cells (Fig. 3) and marked reduction in the expression of caspase- 3 and Bax (Fig. 4).

GYY4137 attenuates the VC-induced inflammatory response. The expression of TNF- $\alpha$, IL-1 $\beta$, Bax and cleaved caspase- 3 was significantly higher in group $\mathrm{C}$ than in groups $\mathrm{A}$ and $\mathrm{B}$ 

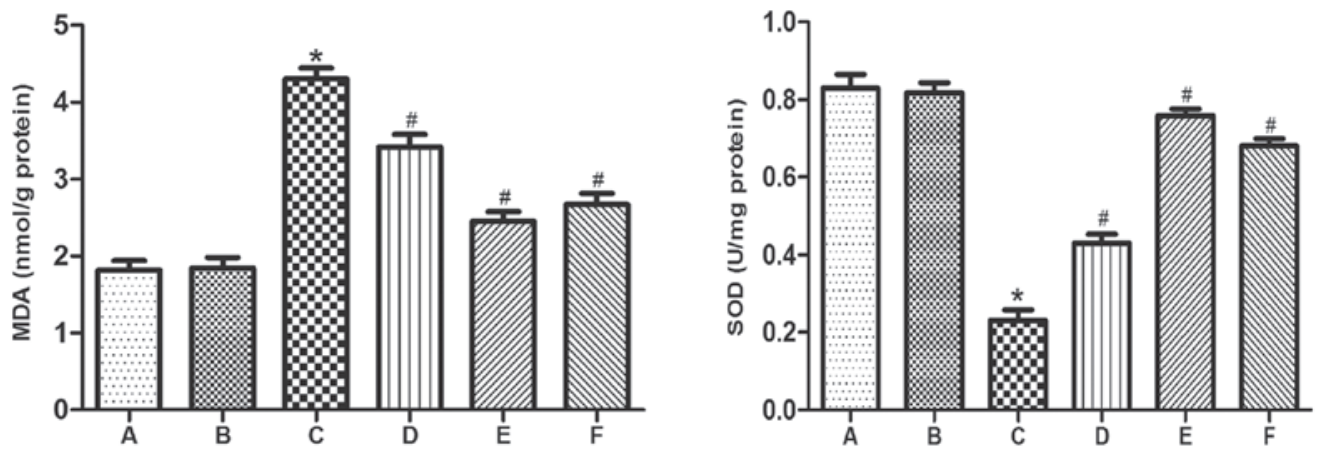

Figure 2. MDA content and SOD activity in the 6 groups. (A) Control group, (B) sham group, (C) VC group, (D) VC+5 mg/kg/day GYY4137 group, (E) VC+10 mg/kg/day GYY4137 group, (F) VC+20 mg/kg/day GYY4137 group. " $\mathrm{P}<0.05$ vs. group A; ${ }^{2} \mathrm{P}<0.05$ vs. group C. VC, varicocele; GYY4127, Morpholin-4-ium 4 methoxyphenyl (morpholino) phosphonodithioate; MDA, malondialdehyde; SOD, superoxide dismutase.
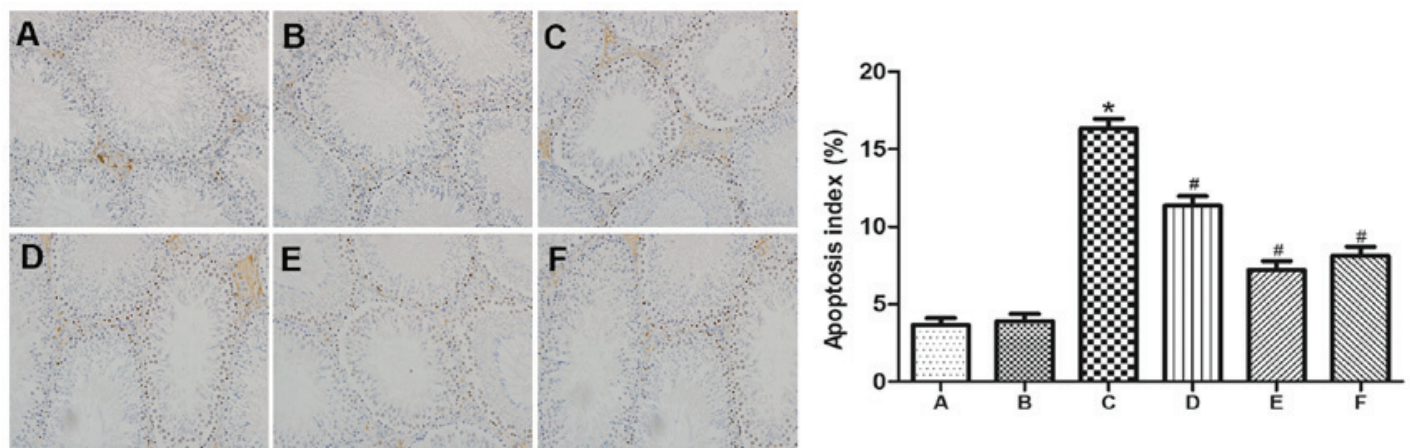

Figure 3. Apoptosis index in the 6 groups. (A) Control group, (B) sham group, (C) VC group, (D) VC+5 mg/kg/day GYY4137 group, (E) VC+10 mg/kg/day GYY4137 group, (F) VC+20 mg/kg/day GYY4137 group. Magnification, x200. " $\mathrm{P}<0.05$ vs. group A; "P<0.05 vs. group C. VC, varicocele; GYY4127, Morpholin-4-ium 4 methoxyphenyl (morpholino) phosphonodithioate.

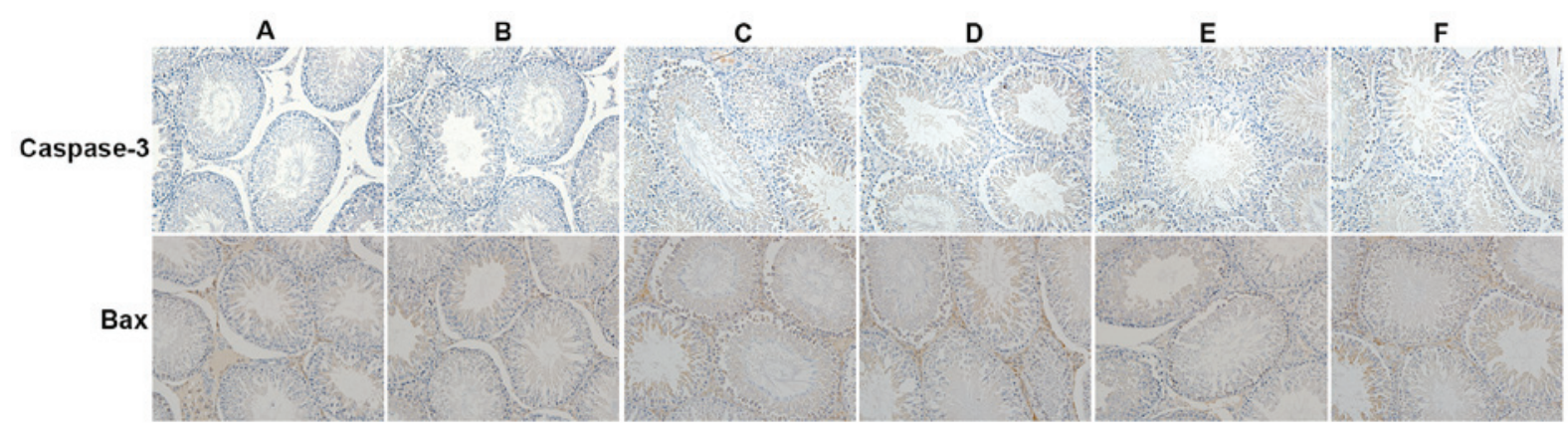

Figure 4. Immunohistochemistry was performed to evaluate the expression of Bax and caspase-3. (A) Control group, (B) sham group, (C) VC group, (D) VC+5 mg/kg/day GYY4137 group, (E) VC+10 mg/kg/day GYY4137 group, (F) VC+20 mg/kg/day GYY4137 group. Magnification, x200. VC, varicocele; GYY4127, Morpholin-4-ium 4 methoxyphenyl (morpholino) phosphonodithioate.

(Figs. 5 and 6). However, administration of GYY4137 significantly decreased the expression of TNF- $\alpha$, IL-1 $\beta$, Bax and cleaved caspase- 3 in groups D, E and F compared with group C. The results demonstrated that GYY4137 was most effective at reducing the expression of inflammation-related genes at a dose of $10 \mathrm{mg} / \mathrm{kg} /$ day (Figs. 5 and 6).

\section{Discussion}

$\mathrm{VC}$ is described as the abnormal expansion, elongation and tortuosity of the spermatic vein and is considered to be the primary cause of infertility in adolescent males (1). The incidence of $\mathrm{VC}$ in the general population is $\sim 15 \%$, however, in infertile men it is much higher, at $35 \%$ (16). Nevertheless, the mechanism of testicular dysfunction and infertility associated with $\mathrm{VC}$ remains unclear, therefore, a number of studies investigating the pathophysiology of this condition have been performed. GYY4137 is a novel $\mathrm{H}_{2}$ S-releasing molecule and induces a wide range of pharmacological effects, including antioxidant, anti-apoptosis, anti-inflammatory and immune-modulating activities, in a wide range of different organs (17-19). The current study initially evaluated the effect of GYY4137 on the histological changes that occur in the testes of rats with a VC. The results demonstrated that VC 

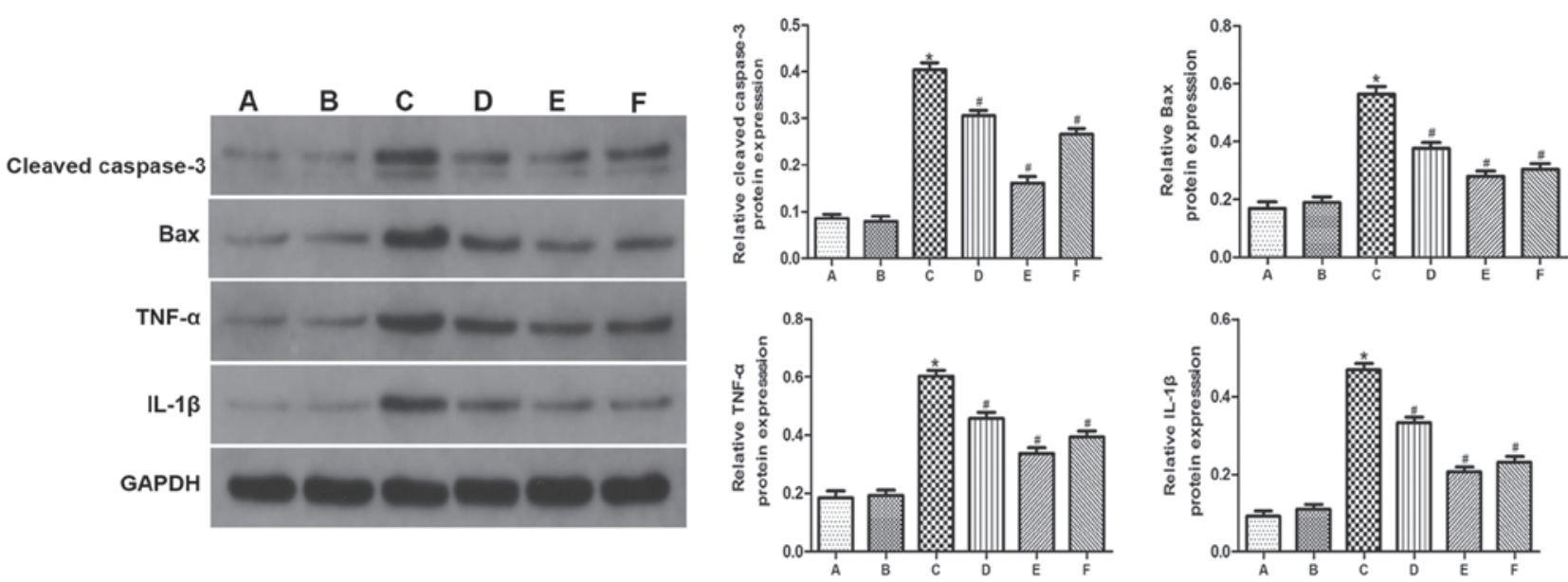

Figure 5. The expression of Bax, cleaved caspase-3, TNF- $\alpha$ and IL-1 $\beta$ was detected by western blotting. GAPDH was as a loading control. (A) Control group, (B) sham group, (C) VC group, (D) VC+5 mg/kg/day GYY4137 group, (E) VC+10 mg/kg/day GYY4137 group, (F) VC+20 mg/kg/day GYY4137 group. "P<0.05 vs. group A; "P<0.05 vs. group C. VC, varicocele; GYY4127, Morpholin-4-ium 4 methoxyphenyl (morpholino) phosphonodithioate; TNF- $\alpha$, tumor necrosis factor $\alpha$; IL-1 $\beta$, interleukin $1 \beta$.
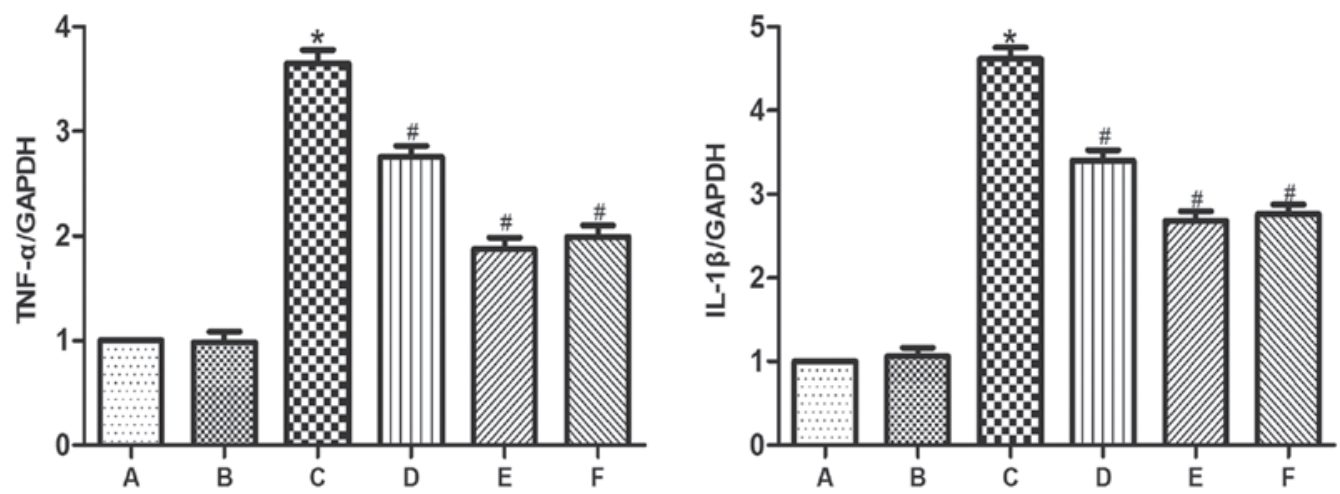

Figure 6. Reverse transcription-quantitative polymerase chain reaction was performed in testes tissues. The expression of TNF- $\alpha$ and IL-1 $\beta$ relative to GAPDH was determined. (A) Control group, (B) sham group, (C) VC group, (D) VC $+5 \mathrm{mg} / \mathrm{kg} / \mathrm{day}$ GYY4137 group, (E) VC+10 mg/kg/day GYY4137 group, (F) VC+20 mg/kg/day GYY4137 group. "P<0.05 vs. group A; "P<0.05 vs. group C. VC, varicocele; GYY4127, Morpholin-4-ium 4 methoxyphenyl (morpholino) phosphonodithioate; TNF- $\alpha$, tumor necrosis factor $\alpha$; IL- $1 \beta$, interleukin $1 \beta$.

severely damages testicular spermatogenic function, in accordance with the results of previous studies $(20,21)$. However, different doses of GYY4137 ameliorated histological injury to different extents and the ideal effect was induced following the administration of $10 \mathrm{mg} / \mathrm{kg} /$ day GYY4137 in experimental rats with a $\mathrm{VC}$.

Oxidative stress is a result of the accumulation of ROS in tissues, which leads to the deregulation of antioxidant mechanisms (22). Under physiological situations, the accumulation of ROS is maintained at a low level throughout the antioxidant defence system and has specific regulatory effects on cell growth, development, differentiation and death. However, in pathological circumstances, including ischemia and hypoxia, the overproduction of ROS can result in the oxidation of cellular membrane proteins, lipids and DNA to cause a range of cellular dysfunction and cell death $(22,23)$. Mammalian testes are highly sensitive to oxidative stress and the pathology of VC is closely associated with the overproduction of ROS (24). MDA, a major product of lipid peroxidative decomposition generated by ROS, is typically used to evaluate the extent of cellular damage under conditions of oxidative stress (25). SOD is a critical component in the processes of cell growth, differentiation and protection, and has the ability to convert a superoxide anion radical into hydrogen peroxide; its activity reflects the scavenging capacity of ROS in organisms (26). It has been demonstrated that $\mathrm{H}_{2} \mathrm{~S}$ serves a key role in reducing ROS production and has therapeutic potential in preventing oxidative stress damage in different types of organs (27). In the current study, treatment with the novel $\mathrm{H}_{2} \mathrm{~S}$ donor, GYY4137, alleviated oxidative stress in $\mathrm{VC}$ testes by reducing the expression of MDA and increasing the expression of SOD.

Apoptosis is a normal physiological phenomenon that serves an important role in maintaining homeostasis during spermatogenesis (28). However, the pathological process of VC usually leads to widespread spermatogenic cell apoptosis, which may induce further testicular dysfunction and cause infertility (29). Zheng et al (30) demonstrated that the incidence of apoptosis in the ipsilateral testis was greatly raised in a rat model of VC and that there was a positive correlation between the extent of apoptosis and injury duration. It has been demonstrated that the process of apoptosis is regulated by gene expression and its 
activation may stimulate the degradation of cellular substrates and participate in a variety of pathogeneses, including acting as a functional obstacle of spermatogenesis and decreasing sperm motility and DNA levels (31). Furthermore, Bax may promote the release of cytochrome $c$ from the mitochondria to induce apoptosis (32). Mostafa et al (33) indicated that the expression of Bax was significantly increased in infertile men with a VC. Additionally, caspase-3 is an inactive zymogen among the cysteine proteases and the convergence point of multiple apoptosis-stimulating signals. Its activation is a sign of an irreversible commitment to cellular apoptosis (30). It has been demonstrated in an androgen-deficiency model that the decrease in testosterone induced by spermatogenic cell apoptosis in testicular tissue is caspase-3 dependent, indicating that caspase- 3 activation induces spermatogenic cell apoptosis (34). Furthermore, it has been demonstrated that exogenous $\mathrm{H}_{2} \mathrm{~S}$ inhibits the activation of caspase- 3 to induce an anti-apoptotic effect in renal ischemia-reperfusion injury (35). The damage induced by VC has complicates the pathological course and inflammation has been recognized as an important factor in the onset and development of VC (36). TNF- $\alpha$ and IL-1 $\beta$ are key inflammatory cytokines involved in the pathological process of VC. Germ cell-derived TNF- $\alpha$ increases tissue damage and the inflammatory response and may control physiological spermatogenic cell apoptosis by regulating Fas ligand levels (37). IL-1 $\beta$ is an immune-derived cytokine and promotes its own secretion under ischemia and hypoxia and the increased expression of IL- $1 \beta$ induces detrimental effects in the testes of infertile males with VC $(38,39)$. In the present study, it was hypothesised that GYY4137 inhibits the expression of Bax, caspase-3, TNF- $\alpha$ and IL-1 $\beta$, thereby alleviating apoptosis and inflammatory responses in a rat model of VC.

In conclusion, the present study demonstrated that GYY4137 attenuates testicular dysfunction by alleviating oxidative stress, inflammation and apoptosis in a rat model of VC. GYY4137 was most effective at attenuating this dysfunction at a dose of $10 \mathrm{mg} / \mathrm{kg} /$ day. However, the specific underlying protective mechanisms of GYY4137 in VC remain unknown and further studies are required.

\section{References}

1. Chan CC, Sun GH, Shui HA and Wu GJ: Differential spermatozoal protein expression profiles in men with varicocele compared to control subjects: Upregulation of heat shock proteins 70 and 90 in varicocele. Urology 81: 1379.e1-e8, 2013.

2. Fretz PC and Sandlow JI: Varicocele: Current concepts in pathophysiology, diagnosis, and treatment. Urol Clin North Am 29: 921-937, 2002.

3. Fazlioglu A, Yilmaz I, Mete O, Kurtulus F, Parlakkilic O, Güctas $\mathrm{O}$ and Cek $\mathrm{M}$ : The effect of varicocele repair on experimental varicocele-induced testicular germ cell apoptosis. J Androl 29: 29-34, 2008.

4. Khosravanian H, Razi M, Farokhi F and Khosravanian N: Simultaneous administration of dexamethasone and vitamin $\mathrm{E}$ reversed experimental varicocele-induced impact in testicular tissue in rats; correlation with Hsp70-2 chaperone expression. Int Braz J Urol 41: 773-790, 2015.

5. Kimura $\mathrm{H}$ : The physiological role of hydrogen sulfide and beyond. Nitric Oxide 41: 4-10, 2014.

6. Lobb I, Zhu J, Liu W, Haig A, Lan Z and Sener A: Hydrogen sulfide treatment ameliorates long-term renal dysfunction resulting from prolonged warm renal ischemia-reperfusion injury. Can Urol Assoc J 8: E413-E418, 2014
7. Li L, Bhatia M and Moore PK: Hydrogen sulphide-a novel mediator of inflammation? Curr Opin Pharmacol 6: 125-129, 2006.

8. Li G, Xie ZZ, Chua JM, Wong PC and Bian J: Hydrogen sulfide protects testicular germ cells against heat-induced injury. Nitric Oxide 46: 165-171, 2015.

9. Liu Z, Han Y, Li L, Lu H, Meng G, Li X, Shirhan M, Peh MT, Xie L, Zhou S, et al: The hydrogen sulfide donor, GYY4137, exhibits anti-atherosclerotic activity in high fat fed apolipoprotein E(-/-) mice. Br J Pharmacol 169: 1795-1809, 2013.

10. Li L, Whiteman M, Guan YY, Neo KL, Cheng Y, Lee SW, Zhao Y, Baskar R, Tan CH and Moore PK: Characterization of a novel, water-soluble hydrogen sulfide-releasing molecule (GYY4137): New insights into the biology of hydrogen sulfide. Circulation 117: 2351-2360, 2008.

11. Meng G, Wang J, Xiao Y, Bai W, Xie L, Shan L, Moore PK and Ji Y: GYY4137 protects against myocardial ischemia and reperfusion injury by attenuating oxidative stress and apoptosis in rats. J Biomed Res 29: 203-213, 2015.

12. Tang B, Ma L, Yao X, Tan G, Han P, Yu T, Liu B and Sun X: Hydrogen sulfide ameliorates acute lung injury induced by infrarenal aortic cross-clamping by inhibiting inflammation and angiopoietin 2 release. J Vasc Surg 65: 501-508.e1, 2017.

13. U.S. National Institutes of Health: Laboratory animal welfare: Public Health Service policy on humane care and use of laboratory animals by awardee institutions; notice. Fed Regist 50: 19584-19585, 1985.

14. Turner TT: The study of varicocele through the use of animal models. Hum Reprod Update 7: 78-84, 2001.

15. Rao X, Huang X, Zhou Z and Lin X: An improvement of the $2^{\wedge}$ (-delta delta CT) method for quantitative real-time polymerase chain reaction data analysis. Biostat Bioinforma Biomath 3: 71-85, 2013.

16. Kilinç F, Kayaselcuk F, Aygun C, Guvel S, Egilmez T and Ozkardes H: Experimental varicocele induces hypoxia inducible factor-1alpha, vascular endothelial growth factor expression and angiogenesis in the rat testis. J Urol 172: 1188-1191, 2004.

17. Rose P, Dymock BW and Moore PK: GYY4137, a novel water-soluble, H2S-releasing molecule. Methods Enzymol 554: 143-167, 2015.

18. Lee ZW, Zhou J, Chen CS, Zhao Y, Tan CH, Li L, Moore PK and Deng LW: The slow-releasing hydrogen sulfide donor, GYY4137, exhibits novel anti-cancer effects in vitro and in vivo. PLoS One 6: e21077, 2011

19. Yuan S, Shen X and Kevil CG: Beyond a Gasotransmitter: Hydrogen sulfide and polysulfide in cardiovascular health and immune response. Antioxid Redox Signal 27: 634-653, 2017.

20. Pastuszak AW and Wang R: Varicocele and testicular function. Asian J Androl 17: 659-667, 2015.

21. Sakamoto H and Ogawa Y: Does a clinical varicocele influence the relationship between testicular volume by ultrasound and testicular function in patients with infertility? Fertil Steril 92: 1632-1637, 2009

22. Turkmen S, Mentese A, Karaguzel E, Karaca Y, Kucuk A Uzun A, Yulug E and Turedi S: A comparison of the effects of $\mathrm{N}$-acetylcysteine and ethyl pyruvate on experimental testicular ischemia-reperfusion injury. Fertil Steril 98: 626-631, 2012.

23. Southorn PA and Powis G: Free radicals in medicine. I. Chemical nature and biologic reactions. Mayo Clin Proc 63: 381-389, 1988.

24. Liang M, Wen J, Dong Q, Zhao LG and Shi BK: Testicular hypofunction caused by activating p53 expression induced by reactive oxygen species in varicocele rats. Andrologia 47: 1175-1182, 2015.

25. Dogan F, Armagan A, Oksay T, Akman T, Aylak F and Bas E: Impact of micronised purified flavonoid fraction on increased malondialdehyde and decreased metalloproteinase- 2 and metalloproteinase-9 levels in varicocele: Outcome of an experimentally induced varicocele. Andrologia 46: 380-385, 2014

26. Sakamoto Y, Ishikawa T, Kondo Y, Yamaguchi K and Fujisawa M: The assessment of oxidative stress in infertile patients with varicocele. Bju Int 101: 1547-1552, 2008.

27. Chen CQ, Xin H and Zhu YZ: Hydrogen sulfide: Third gaseous transmitter, but with great pharmacological potential. Acta Pharmacol Sin 28: 1709-1716, 2007.

28. Vaux DL and Korsmeyer SJ: Cell death in development. Cell 96: 245-254, 1999

29. Tanaka H, Fujisawa M, Tanaka H, Okada H and Kamidono S: Apoptosis-related proteins in the testes of infertile men with varicocele. Bju Int 89: 905-909, 2002.

30. Zheng Y, Zhang X, Zhou J, Cheng F and Zhou B: Effects on the ipsilateral testis during progression of experimental varicocele in rat. Med Sci Monit 14: BR122-BR126, 2008. 
31. Arnoult D, Parone P, Martinou JC, Antonsson B, Estaquier J and Ameisen JC: Mitochondrial release of apoptosis-inducing factor occurs downstream of cytochrome c release in response to several proapoptotic stimuli. J Cell Biol 159: 923-929, 2002.

32. Liang H, Yu F, Tong Z, Yuan B and Wang C: Effect of ischemia post-conditioning on skeletal muscle oxidative injury, mTOR, Bax, Bcl-2 proteins expression, and HIF- $1 \alpha / \beta$-actin mRNA, IL- $6 / \beta$-actin mRNA and caveolin-3/ $\beta$-actin mRNA expression in ischemia-reperfusion rabbits. Mol Biol Rep 40: 507-514, 2013

33. Mostafa T, Rashed L, Nabil N and Amin R: Seminal BAX and BCL2 gene and protein expressions in infertile men with varicocele. Urology 84: 590-595, 2014

34. Kim JM, Ghosh SR, Weil AC and Zirkin BR: Caspase-3 and caspase-activated deoxyribonuclease are associated with testicular germ cell apoptosis resulting from reduced intratesticular testosterone. Endocrinology 142: 3809-3816, 2001.

35. Bos EM, Leuvenink HG, Snijder PM, Kloosterhuis NJ, Hillebrands JL,Leemans JC,Florquin S and van Goor H:Hydrogen sulfide-induced hypometabolism prevents renal ischemia/ reperfusion injury. J Am Soc Nephrol 20: 1901-1905, 2009.

36. Zeinali M, Hadian Amree A, Khorramdelazad H, Karami H and Abedinzadeh M: Inflammatory and anti-inflammatory cytokines in the seminal plasma of infertile men suffering from varicocele. Andrologia: 49, 2016.
37. Pentikäinen V, Erkkila K, Suomalainen L, Otala M, Pentikäinen MO, Parvinen $M$ and Dunkel L: TNFalpha down-regulates the Fas ligand and inhibits germ cell apoptosis in the human testis. J Clin Endocrinol Metab 86: 4480-4488, 2001.

38. Sahin Z, Celik-Ozenci C, Akkoyunlu G, Korgun ET, Acar N, Erdogru T, Demir R and Ustunel I: Increased expression of interleukin-1alpha and interleukin-1beta is associated with experimental varicocele. Fertil Steril 85 (Suppl 1): S1265-S1275, 2006.

39. Wang Y, Ge P and Zhu Y: TLR2 and TLR4 in the brain injury caused by cerebral ischemia and reperfusion. Mediators Inflamm 2013: 124614, 2013.

This work is licensed under a Creative Commons Attribution-NonCommercial-NoDerivatives 4.0 International (CC BY-NC-ND 4.0) License. 